\title{
Early mobilization in the pediatric intensive care unit
}

\author{
Tracie C. Walker ${ }^{1}$, Sapna R. Kudchadkar ${ }^{1,2,3}$ \\ ${ }^{1}$ Department of Anesthesiology \& Critical Care Medicine, Johns Hopkins University School of Medicine, Charlotte R. Bloomberg Children's \\ Center, Baltimore, MD, USA; ${ }^{2}$ Department of Pediatrics, ${ }^{3}$ Department of Physical Medicine \& Rehabilitation, Johns Hopkins University School of \\ Medicine, Baltimore, MD, USA \\ Contributions: (I) Conception and design: None; (II) Administrative support: None; (III) Provision of study materials or patients: None; (IV) \\ Collection and assembly of data: None; (V) Data analysis and interpretation: None; (VI) Manuscript writing: All authors; (VII) Final approval of \\ manuscript: All authors. \\ Correspondence to: Sapna R. Kudchadkar, MD, PhD. Department of Anesthesiology \& Critical Care Medicine, Johns Hopkins University School of \\ Medicine, Charlotte R. Bloomberg Children’s Center, 1800 Orleans Street, Suite 6318B, Baltimore, MD, USA. Email: sapna@jhmi.edu.
}

\begin{abstract}
Survivors of critical illness often experience multiple morbidities that start in the intensive care unit and impact their quality of life after discharge. Reduced physical function, cognitive decline, feeding disorders, and psychological stress are just a few of the potential complications. Many of these morbidities can lead to a reduced quality of life and lifelong impediments. Early mobilization, an intervention that is intended to maintain or restore musculoskeletal strength in the critically ill, has the potential to also yield positive psychological and cognitive benefits. In adults, early mobilization has been shown to be safe, decrease the incidence of delirium, and decrease length of stay. Early mobilization of the pediatric critically ill patient is still a novel topic with a growing body of research. This article will review the current literature on early mobilization of the pediatric critically ill patient.
\end{abstract}

Keywords: Critical illness; early mobilization; pediatrics; rehabilitation

Submitted Aug 05, 2018. Accepted for publication Sep 04, 2018.

doi: $10.21037 /$ tp.2018.09.02

View this article at: http://dx.doi.org/10.21037/tp.2018.09.02

\section{Introduction}

Mortality in the pediatric intensive care unit (PICU) has decreased considerably in the past 10 years but has been exchanged for an increase in PICU-acquired morbidities (1). Emerging literature suggests that adult and pediatric survivors of critical illness experience significant physical, psychosocial, and cognitive morbidities, leading to delayed recovery, functional impairments, and reduced quality of life $(2,3)$. Intensive care unit (ICU) Liberation, a movement that focuses on allowing critically ill patients to be as safely awake, interactive, and mobile as possible, aims to prevent these morbidities (4). Historically, critically ill children were sedated and immobile because of concerns for safety. However, thanks to the ICU Liberation movement, this culture is transforming.

The ABCDEF bundle (Figure 1) is an evidence-based guideline formulated by the ICU Liberation Collaborative, which aims to liberate patients from the iatrogenic aspects of critical illness that threaten a person's self-worth or dignity (5). ICU-acquired weakness, delirium, pain, and agitation are some of these crippling effects seen in our patients. As technological advancements have improved survivability, the recognition of post-ICU syndrome has emerged. The ICU Liberation Collaborative, a large-scale quality improvement project, began by implementing the pain, agitation, and delirium guidelines in in 76 ICUs across the nation, including 9 pediatric units. The ABCDEF bundle was then constructed to advise the ICU team on daily care that they can provide to reduce symptoms of these three conditions. The building blocks of the bundle were obtained from randomized control trials in adults, which showed that these proactive interventions reduced the burden of pain, agitation, or delirium (6-10). The components of the bundle are assessment, prevention and management of pain; both spontaneous awakening 


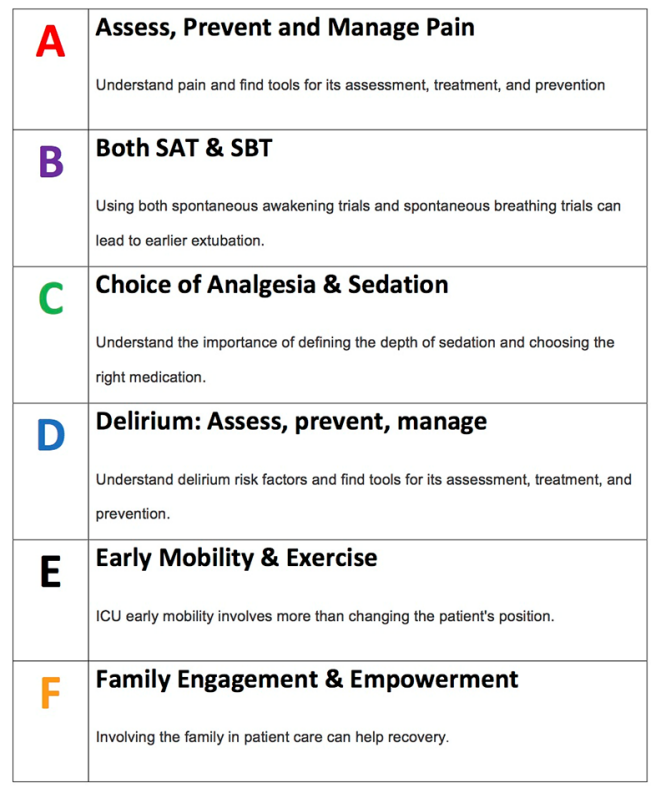

Figure 1 Framework of the ABCDEF bundle. SAT, spontaneous awakening trial; SBT, spontaneous breathing trial.

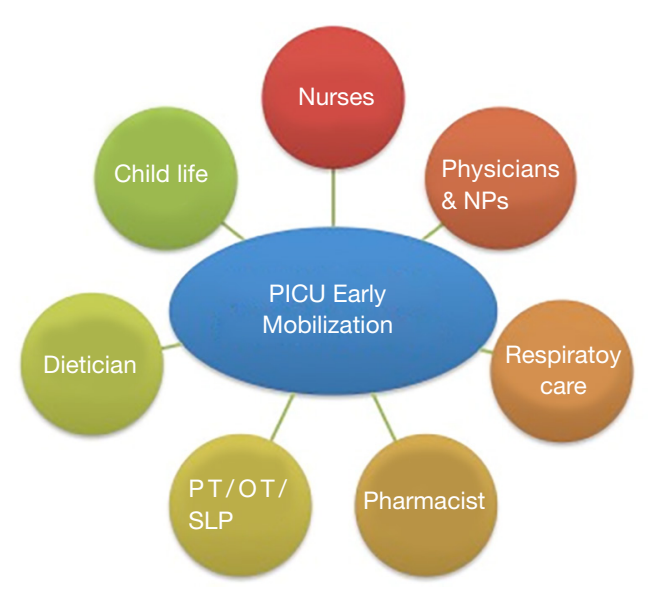

Figure 2 Interdisciplinary stakeholders integral to success of early mobility program creation and implementation in the PICU. PICU, pediatric intensive care unit; NP, nurse practitioner.

and spontaneous breathing trials; choice of sedation and analgesia; delirium assessment, prevention and management; early mobility and exercise; and Family engagement and empowerment.

Early mobilization, a key aspect of the ICU Liberation ABCDEF bundle, is gaining attention in both pediatric and adult critically ill populations. In adults, studies have shown that ICU-based early mobilization is feasible and safe, improves functional outcomes, and is cost effective (6,11-13). Research on mobilization in the PICU is only recently emerging and consists primarily of data from surveys and quality improvement projects. The objective of this review is to summarize data on the safety and feasibility of early mobilization in the PICU, describe outcomes of early rehabilitation in the pediatric critically ill patient, and highlight knowledge gaps and avenues for future research.

\section{Early mobilization: definition, design, and implementation}

Like in the adult ICU, early mobilization in the PICU has no standard definition at this time, with many articles citing differing therapies, varying levels of activity, and variable initiation times. In general, early mobilization is defined as clinically safe and developmentally appropriate rehabilitation exercises of varying degrees initiated within the first 48 to 72 hours of ICU admission $(14,15)$.

Given the heterogeneity of patients' chronological ages, developmental stages, and range of diagnoses, generating a standard rehabilitation plan in the PICU is challenging. Some centers have developed customized mobilization pathways based on a patient's clinical stability and mechanical support, with increasing levels of rehabilitation as the patient's clinical status improves. PICU Up!, a quality improvement project by Wieczorek et al., is a system of stratified rehabilitation levels based on the patient's clinical status that encompasses a spectrum of movement, from passive range of motion activities for the most critically ill patients to ambulation if appropriate (16). Unique modalities of rehabilitation techniques have also been described, such as cycle ergometry (in-bed cycling) (17) and the use of Nintendo Wii for virtual reality exercises $(18,19)$.

The feasibility of implementing early mobilization programs in the PICU has been shown in a variety of pediatric critically ill populations, including cardiac, medical, neurological and post-surgical patients (20-22). Gaining support and assistance from a multidisciplinary team has been universally described as critical to the success of a program (23). Disciplines integral to the successful creation and implementation of PICU early mobilization programs include PICU physicians and nurse practitioners; therapists from divisions such as respiratory therapy, physical therapy, occupational therapy, and speech therapy; nursing staff, including nursing leadership and bedside nurses; ancillary staff from child life; and representatives from family engagement (Figure 2). As key stakeholders, 
at least one champion from each group plays a critical role in determining barriers and facilitators, as perceived by all staff, and ensuring that PICU staff collectively feel engaged in the process of changing the mobility culture.

The implementation and consistency of establishing an early mobilization culture has numerous barriers. A recent survey showed that only $3 \%$ of all Canadian physicians and physiotherapists practiced in a facility that has early mobilization guidelines (24). The most common barriers to mobilizing children are lack of physiotherapy resources, lack of practice guidelines, and concerns for patient safety $(14,16,24)$. In a recent publication by Zheng et al. that conveys the results of post-mobilization interviews, both clinicians and parents expressed similar concerns that mobilization was not prioritized early in the ICU stay (25). Clinicians were primarily concerned for the safety of patients, whereas parents were more focused on prognosis of their child's illness. Families' trust in the healthcare team and clinicians' belief in the importance of early mobilization were the most supportive reasons for early mobilization in the PICU population and facilitated patient motivation. That study highlights the importance of family engagement and clinician buy-in to mobilization of our patients. In the PICU Up! quality improvement project, implementation of an early mobilization activity program decreased the immobility caused by procedures or tests. However, the reported barrier of equipment availability increased. This finding suggests that team coordination for mobilization activities was improved and that missing specialized equipment became more apparent as mobilization was considered for patients previously deemed ineligible.

\section{Safety and feasibility}

Mobilization of critically ill children, many with central lines, endotracheal tubes, and other life-saving devices, is associated with potential risks and complications. Hence, concerns about safety often guide staff perceptions about PICU mobility. These complications may include, but are not limited to, hemodynamic instability, accidental tube or line dislodgement, falls, pain, and anxiety. However, multiple studies have reported that early mobilization of critically ill children is safe when appropriate precautions are taken and levels of mobility are suitable for the level of acuity. The wEECYCLE trial randomized patients to receive in-bed cycling or standard physiotherapy and showed no adverse events in any of the patients in the cycling arm (17). Abdulsatar et al. reported no adverse events related to the use of Nintendo Wii boxing as an exercise modality (18). The large quality improvement initiative PICU Up!, which included 200 patients, showed no associated adverse events with the use of a stratified early rehabilitation program (16). Studies in specialized populations, including children who require ventricular assist devices and postoperative otolaryngology patients, also have failed to show an increase in adverse events after implementation of rehabilitation programs $(20,21)$.

\section{Outcomes}

The literature contains little data on the outcomes of early mobilization in the pediatric critically ill, and within the published data, the outcome measures vary substantially. Rehabilitation using Nintendo Wii boxing showed an increase in upper body activity in the intervention group, although there was no significant change in the patients' grip strength (18). A study performed in adults by Andelic et al. showed significantly improved global functioning, measured by the Disability Rating Scale, in those who received early rehabilitation starting in the ICU (26). Compared with patients who received delayed rehabilitation, a larger proportion of the patients who received early rehabilitation were employed full time and lived at home without assistance at 12 months. Jacobs et al. found that children who were allowed a more liberal activity level postoperatively, specifically without physical or pharmacological restraints, had shorter PICU and total hospital lengths of stay (20). Outcome data are not limited to functional outcomes but also include neuropsychological outcomes. A study by Melchers et al. showed that increased sensory stimulation in pediatric patients with severe traumatic brain injury led to improvements in intelligence scores and quality of life at 6 months after ICU discharge (22).

\section{Discussion}

Although the interest in early mobilization of pediatric patients is increasing, large-scale studies are still lacking. Synthesizing the current data suggests that early mobilization is safe, feasible, and can be employed in a variety of pediatric critically ill populations. The few studies that have evaluated functional outcomes have found benefits of early mobilization specifically in those with traumatic brain injury (26). Additionally, a small study that evaluated 
postoperative otolaryngology patients reported improved quality of life and decreased length of hospital stay with early mobilization. However, even given the supportive data published and the reassurance of no significant adverse events with the establishment of increased rehabilitation, many of our PICU patients are remaining immobilized.

What are the first steps to changing a PICU culture of immobility? Although evidence supports the safety, feasibility, and potential effectiveness of early mobilization programs, the biggest hurdle for most PICUs will be where to start. Culture change is challenging, requiring significant time commitment, a multidisciplinary team, and a structured system. The pediatric population also presents unique challenges to implementing any guidelines given the vast diversity of cognitive ability, physical capacity, and medical diagnoses.

Optimizing sedation goes hand in hand with a culture of mobilization to facilitate patient engagement (27). When targeting mobility, it is crucial that frequent sedation assessments and adjustments to sedation medication be made to ensure that a child is as awake as is safely feasible. Sedation medications that minimize iatrogenic effects should be chosen. Using validated assessment tools such as the state behavioral scale, Ramsay scale, or COMFORT scale can standardize the evaluation and prevent unnecessary oversedation. The presence of delirium, an acute and fluctuating state of consciousness, can also significantly hinder mobilization efforts. Frequent screening of all critically ill children for delirium using the Preschool Confusion Assessment Method (psCAM; 6 months to 5 years) the Cornell-Assessment of Pediatric Delirium (CAPD; all ages) or the Pediatric Confusion Assessment Method (pCAM; 5 years and older), can effectively identify both hypoactive and hyperactive delirium $(28,29)$. Promoting sleep, treating pain, and minimizing benzodiazepine use also decrease the incidence of delirium (30-32). Maintaining children in an alert, lucid, and comfortable state during the daytime hours and optimizing restorative sleep at night is an essential first step to breaking down the wall of immobility culture (33).

Change typically should occur in small increments. Evidence has shown that buy-in from all critical care team members is essential to implement a successful acute rehabilitation program (34). Designing clear guidelines for stratified levels of mobilization based on patient acuity is suggested to promote patient safety and prevent adverse events. A care team's positive experience from a mobilization event will be memorable, but a significant adverse event will be unforgettable and potentially cause a decline in any momentum for mobilization. Therefore, choosing the optimal patient for each step in a mobilization program is imperative to continue the momentum for change.

Transforming the PICU culture to one that facilitates early mobilization is a complex and multistep process. Large-scale studies are needed to evaluate the functional and psychological outcomes as well as the financial impact of early rehabilitation in pediatrics. Such studies will assist in the clinical acceptance of early mobilization. Examining the effect of early rehabilitation on the families of pediatric patients would also be of interest, as various reports quote a positive impact (26), and having family support can propel the movement forward. Using a bundled approach that includes sedation minimization, delirium recognition, and family engagement should be the first step in promoting mobilization, followed by designing a structured, tiered mobilization plan that prioritizes patient safety.

\section{Conclusions}

Early mobilization in the PICU is gaining momentum but still lacks large randomized control trials and functional and psychological outcome data. Evidence suggests that mobilization of critically ill children is safe and feasible, but studies are still needed to ascertain both physical and psychological outcomes. Modifying the PICU culture to minimize sedation, recognize delirium, and promote mobilization will improve the survivorship of our critically ill pediatric patients.

\section{Acknowledgements}

The authors would like to acknowledge Claire Levine for her editorial assistance.

\section{Footnote}

Conflicts of Interest: The authors have no conflicts of interest to declare.

\section{References}

1. Pinto NP, Rhinesmith EW, Kim TY, et al. Long-Term function after pediatric critical illness: results from the survivor outcomes study. Pediatr Crit Care Med 2017;18:e122-30.

2. Manning JC, Pinto NP, Rennick JE, et al. Conceptualizing post intensive care syndrome in children-the PICS-p 
framework. Pediatr Crit Care Med 2018;19:298-300.

3. Herrup EA, Wieczorek B, Kudchadkar SR. Characteristics of postintensive care syndrome in survivors of pediatric critical illness: A systematic review. World J Crit Care Med 2017;6:124-34.

4. ICU liberation: Optimizing quality and efficiency of critical care delivery [Internet]. [cited 2018 Mar 30]. Available online: https://www.beckershospitalreview.com/ quality/icu-liberation-optimizing-quality-and-efficiencyof-critical-care-delivery.html

5. Ely EW. The ABCDEF bundle: science and philosophy of how ICU liberation serves patients and families. Crit Care Med 2017;45:321-30.

6. Schweickert WD, Pohlman MC, Pohlman AS, et al. Early physical and occupational therapy in mechanically ventilated, critically ill patients: a randomised controlled trial. Lancet 2009;373:1874-82.

7. Ely EW, Inouye SK, Bernard GR, et al. Delirium in mechanically ventilated patients: validity and reliability of the confusion assessment method for the intensive care unit (CAM-ICU). JAMA 2001;286:2703-10.

8. Ely EW, Baker AM, Dunagan DP, et al. Effect on the duration of mechanical ventilation of identifying patients capable of breathing spontaneously. N Engl J Med 1996;335:1864-9.

9. Khan BA, Fadel WF, Tricker JL, et al. Effectiveness of implementing a wake up and breathe program on sedation and delirium in the ICU. Crit Care Med 2014;42:e791-5.

10. Girard TD, Kress JP, Fuchs BD, et al. Efficacy and safety of a paired sedation and ventilator weaning protocol for mechanically ventilated patients in intensive care (Awakening and Breathing Controlled trial): a randomised controlled trial. Lancet 2008;371:126-34.

11. Burtin C, Clerckx B, Robbeets C, et al. Early exercise in critically ill patients enhances short-term functional recovery. Crit Care Med 2009;37:2499-505.

12. Adler J, Malone D. Early mobilization in the intensive care unit: a systematic review. Cardiopulm Phys Ther J 2012;23:5-13.

13. Bailey P, Thomsen GE, Spuhler VJ, et al. Early activity is feasible and safe in respiratory failure patients. Crit Care Med 2007;35:139-45.

14. Wieczorek B, Burke C, Al-Harbi A, et al. Early mobilization in the pediatric intensive care unit: a systematic review. J Pediatr Intensive Care 2015;2015:129-70.

15. Cameron S, Ball I, Cepinskas G, et al. Early mobilization in the critical care unit: A review of adult and pediatric literature. J Crit Care 2015;30:664-72.

16. Wieczorek B, Ascenzi J, Kim Y, et al. PICU Up!: impact of a quality improvement intervention to promote early mobilization in critically ill children. Pediatr Crit Care Med 2016;17:e559-66.

17. Choong K, Awladthani S, Khawaji A, et al. Early exercise in critically ill youth and children, a preliminary evaluation: the wEECYCLE pilot trial. Pediatr Crit Care Med 2017;18:e546-54.

18. Abdulsatar F, Walker RG, Timmons BW, et al. "Wii-Hab" in critically ill children: a pilot trial. J Pediatr Rehabil Med 2013;6:193-204.

19. Salem Y, Elokda A. Use of virtual reality gaming systems for children who are critically ill. J Pediatr Rehabil Med 2014;7:273-6.

20. Jacobs BR, Salman BA, Cotton RT, et al. Postoperative management of children after single-stage laryngotracheal reconstruction. Crit Care Med 2001;29:164-8.

21. Hollander SA, Hollander AJ, Rizzuto S, et al. An inpatient rehabilitation program utilizing standardized care pathways after paracorporeal ventricular assist device placement in children. J Heart Lung Transplant 2014;33:587-92.

22. Melchers P, Maluck A, Suhr L, et al. An early onset rehabilitation program for children and adolescents after traumatic brain injury (TBI): methods and first results. Restor Neurol Neurosci 1999;14:153-60.

23. Parchem K, Peck A, Tales K. A multidisciplinary approach to equipment use in pediatric patient mobilization. Crit Care Nurs Q 2018;41:330-9.

24. Choong K, Koo KKY, Clark H, et al. Early mobilization in critically ill children: a survey of Canadian practice. Crit Care Med 2013;41:1745-53.

25. Zheng K, Sarti A, Boles S, et al. Impressions of early mobilization of critically ill children-clinician, patient, and family perspectives. Pediatr Crit Care Med 2018;19:e350-7.

26. Andelic N, Bautz-Holter E, Ronning P, et al. Does an early onset and continuous chain of rehabilitation improve the long-term functional outcome of patients with severe traumatic brain injury? J Neurotrauma 2012;29:66-74.

27. Saliski M, Kudchadkar SR. Optimizing sedation management to promote early mobilization for critically ill children. J Pediatr Intensive Care 2015;4:188-93.

28. Silver G, Kearney J, Traube C, et al. Delirium screening anchored in child development: The Cornell Assessment for Pediatric Delirium. Palliat Support Care 2015;13:1005-11.

29. Smith HA, Gangopadhyay M, Goben CM, et al. The 
Preschool Confusion Assessment Method for the ICU: valid and reliable delirium monitoring for critically ill infants and children. Crit Care Med 2016;44:592-600.

30. Kamdar BB, Martin JL, Needham DM, et al. Promoting sleep to improve delirium in the ICU. Crit Care Med 2016;44:2290-1.

31. Mody K, Kaur S, Mauer EA, et al. Benzodiazepines and development of delirium in critically ill children: estimating the causal effect. Crit Care Med 2018;46:1486-91.

Cite this article as: Walker TC, Kudchadkar SR. Early mobilization in the pediatric intensive care unit. Transl Pediatr 2018;7(4):308-313. doi: 10.21037/tp.2018.09.02
32. Traube C, Silver G, Reeder RW, et al. Delirium in critically ill children: an international point prevalence study. Crit Care Med 2017;45:584-90.

33. Kudchadkar SR, Aljohani OA, Punjabi NM. Sleep of critically ill children in the pediatric intensive care unit: a systematic review. Sleep Med Rev 2014;18:103-10.

34. Morris PE, Goad A, Thompson C, et al. Early intensive care unit mobility therapy in the treatment of acute respiratory failure. Crit Care Med 2008;36:2238-43. 